\title{
New CUORICINO results on the way to CUORE
}

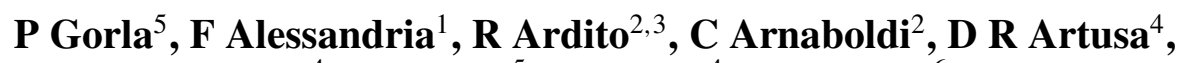
F T Avignone III $^{4}$, M Balata ${ }^{5}$, I Bandac ${ }^{4}$, M Barucci ${ }^{6}$, J W Beeman ${ }^{7}$, F Bellini ${ }^{8}$, C Brofferio ${ }^{2}$, C Bucci $^{5}$, S Capelli ${ }^{2}$, L Carbone $^{2}$, S Cebrian $^{9}$, M Clemenza $^{2}$, C Cosmelli ${ }^{8}$, O Cremonesi $^{2}$, R J Creswick ${ }^{4}$, I Dafinei ${ }^{8}$, S Di Domizio ${ }^{10}$, M Diemoz ${ }^{8}$, M J Dolinski ${ }^{11}$, H A Farach ${ }^{4}$, F Ferroni ${ }^{8}$, E Fiorini $^{2}$, S J Freedman ${ }^{7,12}$, C Gargiulo ${ }^{8}$, A Giachero ${ }^{5,10}$,

E Guardincerri ${ }^{10}$, A Giuliani ${ }^{13}$, T D Gutierrez ${ }^{7}$, E E Haller $^{7,16}$, K Heeger $^{7}$, I G Irastorza ${ }^{9}$, E Longo $^{8}$, G Maier $^{3}$, R Maruyama $^{12}$, S Morganti ${ }^{8}, \mathbf{S}$ Nisi $^{5}$, C Nones $^{2}$, E B Norman ${ }^{11}$, A Nucciotti ${ }^{2}$, P Ottonello ${ }^{10}$, M Pallavicini ${ }^{10}$, V Palmieri ${ }^{15}$, M Pavan ${ }^{2}$, M Pedretti ${ }^{13}$, G Pessina ${ }^{2}$, S Pirro ${ }^{2}$, E Previtali ${ }^{2}$, L Risegari ${ }^{6}$, C Rosenfeld ${ }^{4}$, S Sangiorgio ${ }^{13}$, M Sisti ${ }^{2}$, A R Smith ${ }^{7}$,

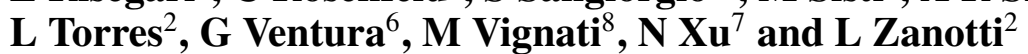

${ }^{1}$ INFN-Sezione di Milano, Milano I-20133, Italy

${ }^{2}$ Dipartimento di Fisica dell'Universitàdi Milano-Bicocca e Sezione di Milano dell'INFN, Milano I-20126, Italy

${ }^{3}$ Dipartimento di Ingegneria Strutturale del Politecnico di Milano, Milano I-20133, Italy

${ }^{4}$ Department of Physics and Astronomy, University of South Carolina, Columbia, SC 29208, USA

${ }^{5}$ Laboratori Nazionali del Gran Sasso, I-67010, Assergi (L'Aquila), Italy

${ }^{6}$ Dipartimento di Fisica dell'Universitàdi Firenze e Sezione di Firenze dell'INFN, Firenze I-50125, Italy

${ }^{7}$ Nuclear Science Division, Lawrence Berkeley National Laboratory, Berkeley, CA 94720, USA

${ }^{8}$ Dipartimento di Fisica dell'Universitàdi Roma La Sapienza e Sezione di Roma dell'INFN,

Roma I-00185, Italy

${ }^{9}$ Laboratorio de Fisica Nuclear y Altas Energias, Universidàd de Zaragoza, 50009 Zaragoza, Spain

${ }^{10}$ Dipartimento di Fisica dell'Universitàdi Genova e Sezione di Genova dell'INFN,

Genova I-16146, Italy

${ }^{11}$ Lawrence Livermore National Laboratory, Livermore, CA 94550, USA

${ }^{12}$ Department of Physics, University of California, Berkeley, CA 94720, USA

${ }^{13}$ Dipartimento di Fisica e Matematica dell'Universitàdell'Insubria e Sezione di Milano dell'INFN, Como I-22100, Italy

${ }^{14}$ Physics Division, Lawrence Berkeley National Laboratory, Berkeley, CA 94720, USA

${ }^{15}$ Laboratori Nazionali di Legnaro, I-35020 Legnaro (Padova), Italy

${ }^{16}$ Department of Materials Science and Mineral Engineering, University of California, Berkeley, CA 94720, USA

E-mail: paolo.gorla@lngs.infn.it

Received 24 June 2006

Accepted for publication 3 July 2006

Published 1 September 2006

Online at stacks.iop.org/PhysScr/T127/49

\begin{abstract}
CUORE is a 0.75 ton experiment to search for neutrinoless double beta decay of $\mathrm{Te}^{130} \mathrm{using}$ $988 \mathrm{TeO}_{2}$ bolometers. It aims at reaching a sensitivity on the effective neutrino mass of the order of few tens of meV. CUORICINO, a single CUORE tower running since 2003, plays an important role as a stand alone experiment and for developing the future CUORE setup. Present results already achieved and studies that are underway are here presented and discussed.
\end{abstract}

PACS number: 23.40

(Some figures in this article are in colour only in the electronic version.) 


\section{Introduction}

The evidence of a neutrino rest mass represents one of the most exciting discoveries in the field of particle physics. The discovery of the neutrinoless double beta decay (0v-DBD), however, will provide not only the ultimate answer about the nature (Dirac or Majorana) of the neutrino, but will also allow a sensitivity on the mass down to a few meV. The use of the bolometric technique offers the unique possibility to investigate different $0 v$-DBD candidates with considerable high energy resolution, needed to separate the $2 v$ contribution from the $0 v$ peak. The CUORE experiment [1], to search $0 \nu$-DBD of ${ }^{130} \mathrm{Te}$, will start its assembling phase in 2007 and it aims to reach a sensitivity on Majorana mass better than $50 \mathrm{meV}$. CUORICINO represent not only the first stage of CUORE, but also the most massive 0v-DBD experiment presently running.

\section{CUORICINO setup}

CUORICINO [2] is an array of 62 crystals of $\mathrm{TeO}_{2}$ with a total active mass of $40.7 \mathrm{~kg}$, that corresponds to a mass of ${ }^{130} \mathrm{Te}$ of $\sim 11 \mathrm{~kg}$. The tower is located inside the cryostat situated in Hall A of Laboratori Nazionali del Gran Sasso (LNGS) of INFN. CUORICINO's 62 crystals are arranged in a tower made by 13 planes (figure 1), 11 of them are filled with four cubes of $5 \mathrm{~cm}$ side while the other two with nine crystals $3 \times 3 \times 6 \mathrm{~cm}^{3}$ each. Four $3 \times 3 \times 6 \mathrm{~cm}^{3}$ crystals are enriched, two with ${ }^{128} \mathrm{Te}, 82.3 \%$ isotopic abundance, and the other two with ${ }^{130} \mathrm{Te}$, isotopic abundance of $75 \%$.

All the materials making up the detector were selected for low contamination with radioactive isotopes. To avoid external vibrations reaching the detectors the tower is mechanically decoupled from the cryostat through a steel spring. In order to shield the materials of the refrigerator from radioactive contaminants, a $1.2 \mathrm{~cm}$ shield of Roman lead with ${ }^{210} \mathrm{~Pb}$ activity of $4 \mathrm{mBq} \mathrm{kg}^{-1}$ [3] is framed around the array to reduce the activity of the thermal shields. The cryostat is externally shielded by means of two layers of lead of $10 \mathrm{~cm}$ minimal thickness each. The background due to environmental neutrons is reduced by a layer of borated polyethylene of $10 \mathrm{~cm}$ minimum thickness. The refrigerator operates inside a Plexiglass anti-radon box flushed with clean $\mathrm{N}_{2}$ and inside a Faraday cage to reduce electromagnetic interferences. Thermal pulses are recorded by neutron transmutation doped Ge thermistors thermally coupled to each crystal. CUORICINO is operated at a temperature of $\sim 8 \mathrm{mK}$ with a spread of $\sim 1 \mathrm{mK}$. The energy calibration is performed before and after each subset of runs, which lasts about a month, by exposing the array to two thoriated tungsten wires inserted in the immediate vicinity of the refrigerator.

\section{Physics results}

The first CUORICINO measurement started in March 2003 and ended in October 2003. After a substantial operation of maintenance in April 2004 the second run of CUORICINO started. The average pulse amplitude is $215 \mu \mathrm{V} \mathrm{MeV}^{-1}$ for the $5 \times 5 \times 5$ crystals and $430 \mu \mathrm{V} \mathrm{MeV}^{-1}$ for the

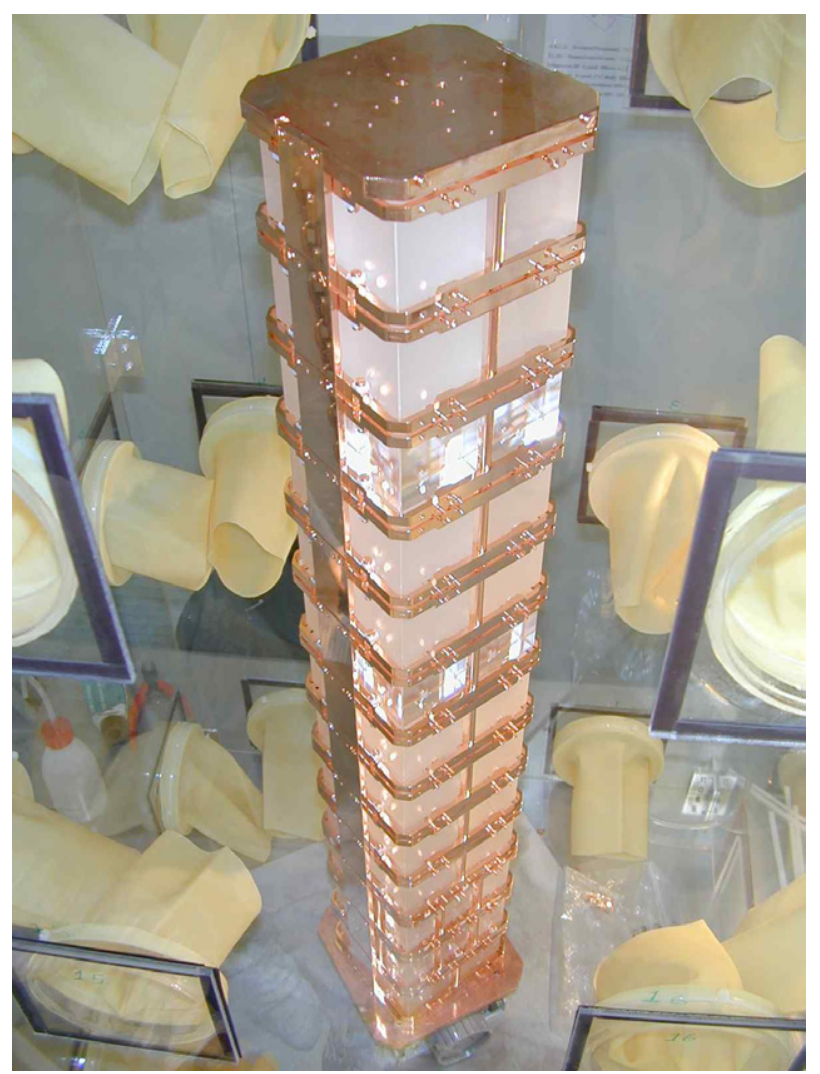

Figure 1. The CUORICINO tower inside a nitrogen-fluxed glove-box, during the final assembly of the detector.

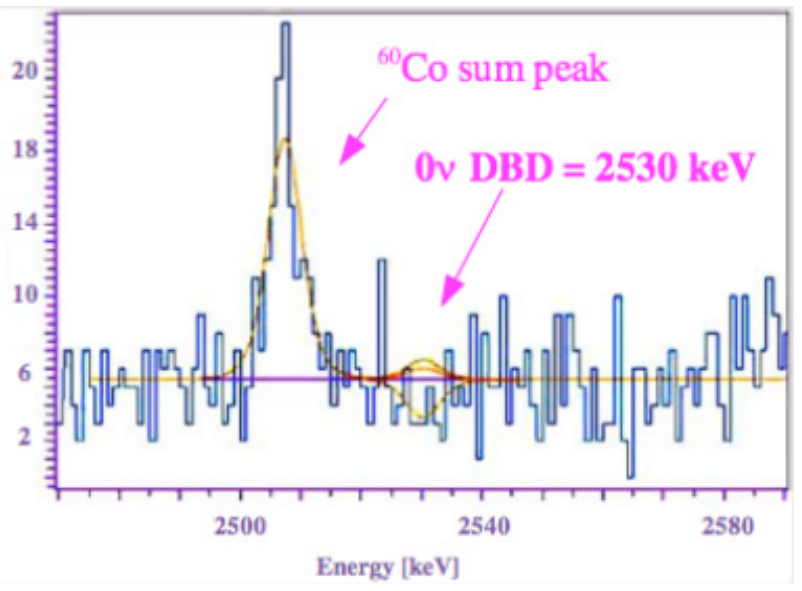

Figure 2. CUORICINO spectrum in the $0 v \mathrm{DBD}$ energy region.

$3 \times 3 \times 6 \mathrm{~cm}^{3}$ crystals. The average resolution FWHM is $7.5 \pm 2.9 \mathrm{keV}$ for the bigger size and $9.6 \pm 3.5 \mathrm{keV}$ for the small size crystals. The duty cycle of the experiment, since August 2004 is $\sim 73 \%$. Discarding the time needed for energy calibration measurement ( 3 days every 3-4 weeks) the total background live time is $63 \%$. The total background spectra collected up to July 2005, corresponding to a total statistic of $8.38 \mathrm{~kg}$ (of ${ }^{130} \mathrm{Te}$ ) year, is presented in figure 2. Apart from the ${ }^{60} \mathrm{Co}$ sum line and the ${ }^{208} \mathrm{Tl}$ line, no other unexpected peak is found near the $2528 \mathrm{keV} 0 \nu \mathrm{DBD}$ region of ${ }^{130} \mathrm{Te}$. The obtained lower limit on the $0 v \mathrm{DBD}$ of ${ }^{130} \mathrm{Te}$ is $2.4 \times 10^{24}$ 


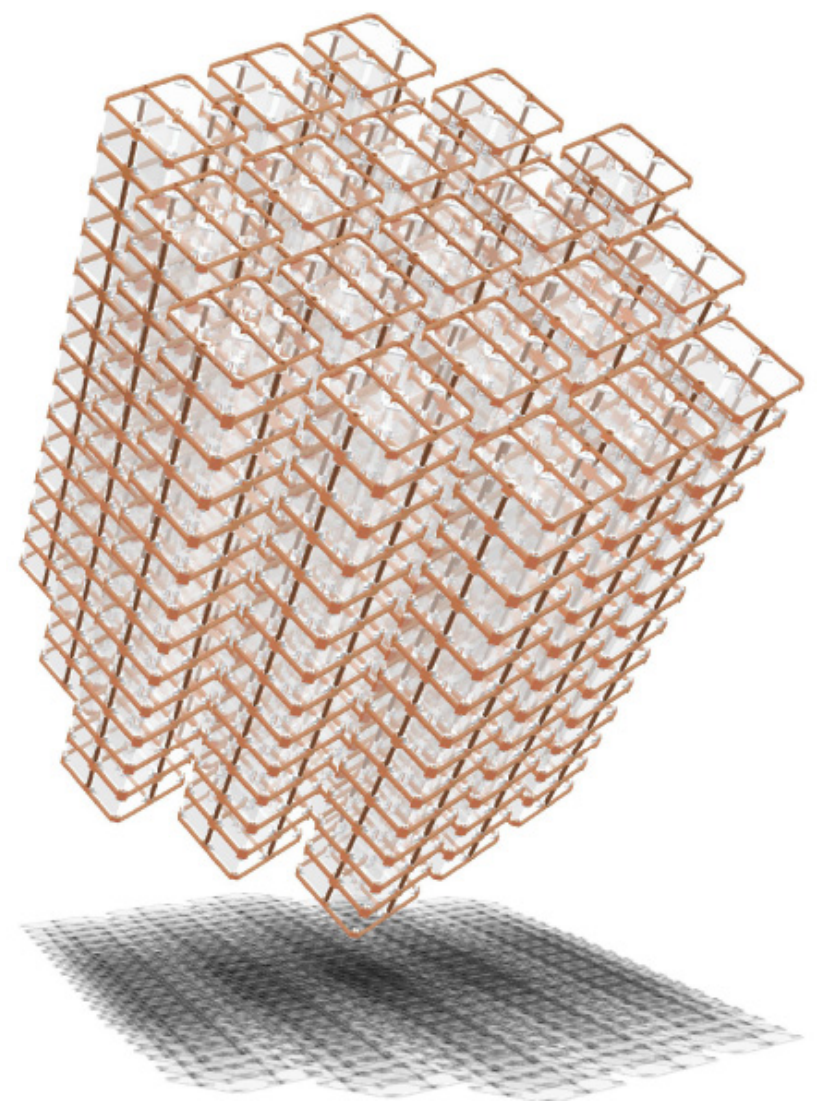

Figure 3. The CUORE detector (cylindrical-shaped) built of 19 CUORICINO-like tower.

year $(90 \% \mathrm{CL})$. This limit leads to a constraint on the electron neutrino effective Majorana mass ranging from 0.18 to $0.97 \mathrm{eV}$, depending on the nuclear matrix elements considered in the computation.

\section{The CUORE experiment}

The CUORE detector will consist of an array of $988 \mathrm{TeO}_{2}$ bolometers arranged in a cylindrical configuration of 19 towers containing 52 crystals each (figure 3), for a total mass of $\sim 741 \mathrm{~kg}$. Each of these towers is a CUORICINOlike detector consisting of 13 modules, four detectors each. Assuming a background of $B=0.01 \mathrm{ckeV}^{-1} \mathrm{~kg}^{-1} \mathrm{yr}^{-1}$, achievable with a slight improvement of the current available material selection and cleaning techniques, and an energy resolution $\Gamma(2.5 \mathrm{MeV})=5 \mathrm{keV}$, we get a sensitivity $S_{0 v}$ on the half life $(90 \% \mathrm{CL})$ of $5.8 \times 10^{25} \sqrt{t}$ years $(4.1 \times$ $10^{25} \sqrt{t}$ years for $\Gamma=10 \mathrm{keV}$ ), which in 5 years of statistics would provide $\left|\left\langle m_{\nu}\right\rangle\right|$ bounds in the range $0.024-0.13 \mathrm{eV}$. However, the R\&D to be carried out in CUORE, if successful, would provide a value of $B \sim 0.001 \mathrm{ckeV}^{-1} \mathrm{~kg}^{-1} \mathrm{yr}^{-1}$, i.e. a detection sensitivity of $S_{0 v} \sim 1.86 \times 10^{26} \sqrt{t}$ years $(1.2 \times$ $10^{26} \sqrt{t}$ years for $\left.\Gamma=10 \mathrm{keV}\right)$, or $\left|\left\langle m_{v}\right\rangle\right|$ bounds in the range $\sim 0.016-0.085 \mathrm{eV}$ in 5 years. $\mathrm{TeO}_{2}$ crystals made with ${ }^{130} \mathrm{Te}$ enriched material have been already operated in MiDBD and CUORICINO, making an enriched CUORE a feasible option. Assuming a $95 \%$ enrichment in ${ }^{130} \mathrm{Te}$ and a background level of $B=0.01 \mathrm{c} \mathrm{keV}^{-1} \mathrm{~kg}^{-1} \mathrm{yr}^{-1}$, the sensitivity would become $S_{0 v} \sim 8.32 \times 10^{26} \sqrt{t}$ years. For an exposure of 5 years, the corresponding $\left|\left\langle m_{\nu}\right\rangle\right|$ bounds would range from 8 to $45 \mathrm{meV}$ depending on the nuclear matrix element calculations.

\section{References}

[1] Arnaboldi C et al 2004 Nucl. Instrum. Methods A $\mathbf{5 1 8} 775$

[2] Arnaboldi C et al 2005 Phys. Rev. Lett. 95142501 (Preprint hep-ex/0501034)

[3] Alessandrello A et al 1998 Nucl. Instrum. Methods B 142163 